NOTICE WARNING CONCERNING COPYRIGHT RESTRICTIONS:

The copyright law of the United States (title 17, U.S. Code) governs the making of photocopies or other reproductions of copyrighted material. Any copying of this document without permission of its author may be prohibited by law. 


\title{
Recognizing Substrings of LR(k) Languages in Linear Time
}

\author{
Joseph Bates Alon Lavie \\ October 1991 \\ CMU-CS-91-188 z
}

\author{
School of Computer Science \\ Carnegie Mellon University \\ Pittsburgh, PA 15213
}

\begin{abstract}
An extended and expanded version of paper by the same title to appear in Proceedings of Principles of Programming Languages, Albuquerque, New Mexico, January 1992 (ACM Press).
\end{abstract}

\begin{abstract}
LR parsing techniques have long been studied as efficient and powerful methods for processing context free languages. A linear time algorithm for recognizing languages representable by $L R(k)$ grammars has long been known. Recognizing substrings of a context-free language is at least as hard as recognizing full strings of the language, as the latter problem easily reduces to the former. In this paper we present a linear time algorithm for recognizing substrings of LR(k) languages, thus showing that the substring recognition problem for these languages is no harder than the full string recognition problem. An interesting data structure, the Forest Structured Stack, allows the algorithm to track all possible parses of a substring without loosing the efficiency of the original LR parser. We present the algorithm, prove its correctness, analyze its complexity, and mention several applications that have been constructed.
\end{abstract}

This research was supported in part by National Science Foundation grant IRI-8858085.

The views and conclusions contained in this document are those of the authors and should not be interpreted as representing the official policies, either expressed or implied, of the NSF or the U.S. government. 


510.7808
$c 28 r$
$91-188$
$c .2$

Keywords: amortization, context-free grammars, parsing 


\section{Introduction}

The problem of recognizing substrings of context-free languages (CFLs) has emerged in many practical applications, in the areas of both formal and natural languages. Given a string $x$, we wish to know whether there exists some string $w$, such that $x$ is a substring of $w$, and $w$ is in the language of a given context-free grammar $G$. The ability to recognize that a given string is not a substring of any sentence in the language allows the early and local detection of syntax errors, without the need to complete a full parse or compilation.

Recognition and parsing algorithms for CFLs have been studied extensively in the last three decades. Valiant [Val75] reduced the problem of recognizing a CFL to that of matrix multiplication, resulting in an indirect algorithm for CFL recognition with the best asymptotic time complexity known. This upper bound on matrix multiplication stands today at $\mathrm{O}\left(n^{2.376}\right)$ [CW87]. However, other algorithms such as Earley's [Ear70] have proven to be much more efficient in practice. The LR parsing techniques have become popular as efficient and powerful methods for parsing CFLs. They were first proposed by Knuth [Knu65] and further developed by Korenjak [Kor69] and DeRemer [DeR69] [DeR71]. LR parsers parse the input bottom-up, scanning the input left to right, producing a right-most derivation. They are deterministic and efficient, being driven by a table of parsing actions pre-compiled from the grammar.

Unfortunately, it is not always possible to construct an LR parser for an arbitrary context-free grammar. However, parsers can be constructed for a large class of grammars, called LR grammars. Lookaheads in the input can help resolve table conflicts that arise in the LR tables constructed for certain grammars, and enable the construction of a deterministic table. Parsers that employ such lookaheads are named $L R(k)$ parsers, and the grammars that may be parsed by them are called LR(k) grammars. A detailed description of the theory behind the various LR parsing techniques, along with formal proofs of the the correctness of the algorithms presented, may be found in Aho and Ullman [AU72]. A more practical description of LR parsers appears in Aho and Ullman [AU77] and Aho Sethi and Ullman [ASU86], and a general survey of LR parsing can be found in Aho and Johnson [AJ74].

Substring recognizers have been considered in several works on recovery from syntax errors. Richter [Ric85] develops a formal method for reporting syntax errors, without attempting to correct them. His method requires a substring recognizer, although no such recognizer is described in his paper. Cormack [Cor89] describes a method for constructing an LR parser that recognizes all substrings of a context-free grammar $G$. This is done by a more complicated construction of the LR parsing tables, appropriate for dealing with substrings. Cormack's construction provides a deterministic parser (free of table conflicts) for only the bounded context class of grammars, which is a class smaller than LR(1). Rekers and Koorn [RK91] propose a substring parsing algorithm for arbitrary context-free grammars based on Tomita's generalized LR parsing algorithm [Tom86]. Although their algorithm has some similarities to the one proposed here, it is not linear, and it's correctness and complexity are not addressed in their paper. Substring recognizers appear to be also useful in the context of parallel and incremental parsing [Lin70] [AD83] [Cel78]. In particular, it has recently been brought to our attention that the seeds to some of the ideas present in our substring recognition algorithm can be traced back to Lindstrom's early work on incremental parsers [Lin70].

The substring recognition problem can be shown to be at least as hard as the full-string recognition problem, as the latter problem is easily reducible to the first in constant time and space. The outline of such a reduction is as follows :

Given a context-free grammar $G$ and an input string $x$, we wish to construct a context-free grammar $G^{\prime}$ and string $w$, such that $x \in L(G)$ iff $w$ is a substring of some $y \in L\left(G^{\prime}\right)$. We modify 
the grammar $G$ by adding the rule $S^{\prime} \rightarrow \$ S \$$, where $S$ is the start symbol of grammar $G$, and ' $\$$ ' is a new terminal symbol, not in the original alphabet of grammar $G$. The non-terminal $S^{\prime}$ becomes the new start symbol of grammar $G^{\prime}$. From the input string $x$ we construct $w=\$ x \$$. The output of the reduction is the pair $\left(G^{\prime}, w\right)$. This reduction is constructible in constant time and space. The details of this reduction's correctness proof are omitted, and may be easily filled in by the reader.

Also, it can be shown that the set of all substrings of a CFL is itself a CFL. Since the set of CFLs is exactly the set of languages accepted by non-determinitic pushdown automata (NPDAs), one easy way to show this is by constructing an NPDA that accepts all substrings of the language of a given context-free grammar. The NPDA constructed for accepting the language of a given context-free grammar (in Greibach normal form) in [HU79] (page 116) can easily be modified to accept all substrings of the language. Thus, the general problem of recognizing substrings is not any harder than that of recognizing full-strings. However, the set of all substrings of an $\operatorname{LR}(\mathrm{k})$ language is not necessarily itself an LR(k) language, therefore a linear time bound for recognizing substrings of $\operatorname{LR}(\mathrm{k})$ languages is not trivial.

In this paper we show that the substring recognition problem for LR(k) grammars is not any harder than the full-string recognition problem. We present an algorithm for the LR(k) substring recognition problem that runs in linear time, which is similar to that of the original LR parsing algorithm [AU72]. While previous substring parsing algorithms such as Cormack's [Cor89] modified the LR parsing tables to accommodate for substring recognition, our algorithm modifies the parsing algorithm itself, while leaving the original LR parsing tables intact. We introduce a data structure, the Forest Structured Stack (FSS), that keeps track of all possible parses of the substring, while preserving the efficiency of the original LR parsing algorithm. The SLR, canonical LR(1) and LALR parser variants differ only in the algorithms that produce the parsing tables from the grammar, and share a common LR parsing algorithm that is controlled by these tables. Since our substring algorithm replaces this run-time parsing algorithm while using the parsing tables "as is", it is equally applicable to all of the above LR variants. The parsing algorithm for canonical $L R(k)$ grammars $(k \geq 2)$ differs slightly from the other variants, in order to account for the extended lookahead into the input. Thus, a slightly different version of our substring algorithm handles canonical $\operatorname{LR}(\mathrm{k})$ grammars.

Section 2 describes the FSS data structure and presents the substring recognition algorithm for LR(1) grammars. In section 3 we prove the correctness of the algorithm. Section 4 analyzes the time complexity of our algorithm. An amortized analysis is used to prove that the algorithm does indeed run in linear time. Section 5 extends the algorithm to the general LR(k) case. Finally, some applications of the algorithm and our conclusions are presented in section 6 .

\section{The Algorithm}

In this section we present our fundamental substring recognition algorithm, appropriate for SLR, canonical LR(1) and LALR parsing tables. These LR parsing variants assume that only the single next input symbol is available to the parser at any point (no further lookaheads). The slightly modified algorithm for canonical LR(k) grammars $(k \geq 2)$ is presented in section 5 . The substring recognition algorithm we describe in this section is denoted by $S S R$. It is a variation of the conventional LR parsing algorithm, denoted by $L R P$. 


\subsection{The Forest Structured Stack}

The Forest Structured Stack (FSS) is a graph, consisting of a set of trees, representing a possibly infinite set of stacks of $L R P$. The nodes of the graph are labeled by states of the LR machine. The edges that connect the state nodes are labeled by grammar symbols. Each path from a root to a leaf corresponds to the top portion of an $L R P$ stack, in which the node at the root of the path represents the state at the top of the stack.

The algorithm simulates the behavior of $L R P$ on all the stacks represented in the FSS, adding nodes in correspondence with actions that push items on the stack (shifts), and removing nodes in correspondence with stack reductions. The tree representation avoids the duplication of stacks which have an identical top part but which differ in content deeper down.

\subsection{An Informal Description of the Algorithm}

The idea behind $S S R$ is to effectively simulate the behavior of $L R P$ on all possible strings of which the input is a suffix. When parsing a string $w$, of which our input string $x=x_{1} x_{2} \cdots x_{n}$ is a suffix, $L R P$ is in some state (at the top of the stack) upon shifting $x_{1}$, the first symbol of $x$. We are interested in all such states and thus we initialize SSR by building a FSS with a distinct single node tree for each state that can be the result of shifting $x_{1}$ according to the pre-compiled action table. Since each single node tree represents all stacks with that state at the top, the initial FSS represents the set of all possible stacks after the shifting of $x_{1}$.

From here on we continue the parsing of $x$ according to each of the FSS trees. SSR performs a series of alternating Reduce and Shift phases, one pair of phases for each input symbol.

During a Reduce Phase, reductions are performed on all trees whose top state indicates that a reduction is to be performed. In LR parsing, reductions remove nodes from the stack. When performed on a tree, they are done on all paths in the tree, starting at the root, to a depth corresponding to the number of symbols on the right-hand side of the rule being reduced.

Reductions are a problem only when they wish to remove nodes deeper than the length of some path in the FSS. This corresponds to a reduction that includes symbols derived from parsing the part of the full string that is prior to $x$. In our algorithm, we refer to such reductions as long reductions, and treat them in a manner somewhat similar to our initialization.

A reduction normally removes the right-hand side of the rule being reduced, and then shifts the non-terminal symbol $A$ of the left-hand side of the rule. The new state at the top of the stack is determined from the goto table, and depends on $A$ and on the state revealed at the top of the stack by the reduction. With long reductions, since only a partial stack exists, this state is not known. Our algorithm determines all such possible states by a lookup in the long reduction goto table. This supplemental table specifies for each possible reduction from a state at the top of the stack, the set of states that may be reached as a result of the shifting of the left-hand side non-terminal of the rule being reduced. The table is easily constructed from the parsing tables prior to run-time. Each of the determined goto states corresponds to at least one full string, the parsing of which would have resulted in that state being at the stack top at this point in the parsing process. It is sufficient at this point to add these states to the FSS as single node trees. Long reductions are performed at most once per state in a Reduce Phase, since a second long reduction from the same top state would produce the same new trees, and thus would be redundant ${ }^{1}$.

\footnotetext{
${ }^{1}$ We believe that we can manage without the long reduction goto table, and simply consider all states that are a result of shifting $A$. This leads to a somewhat simpler implementation, but the proof of correctness appears to be
more difficult in this case.
} 
When the action defined by the table on the root node of a tree is error, the entire tree is discarded. These are trees that correspond to prefix strings of $x$ that cannot be completed to strings in the language. A Reduce Phase terminates when the action indicated by the table, on each of the tree root nodes, is to shift the next input symbol. All the shift operations are done in the consequent Shift Phase of the algorithm.

Upon reaching the end of the input $x$, if the FSS is not empty, we can safely assume that there exists a prefix string $y$ such that the parsing of the string $y x$ by the LR parser would not have caused a parsing error by this point. Properties of $L R P$ guarantee the existence of a suffix $z$, such that $w=y x z$ is accepted. Thus $x$ is confirmed to be a valid substring.

To increase the efficiency of the algorithm, two operations, SUBSUME and CONTRACT, are performed on the FSS structure at appropriate times. When a single node tree is added to the FSS, and the state of the node is identical to that of some other tree root node in the FSS, the larger tree may be deleted from the FSS, since the single node tree represents all stacks of $L R P$ that have that particular state at the top of the stack. This set of stacks necessarily includes all stacks that were represented by the larger tree rooted at a node of the same state. The SUBSUME operation detects such conditions and deletes the larger tree. Long reductions frequently create single node trees that subsume other trees in the FSS.

The CONTRACT operation merges two trees, the roots of which are of the same state, returning a single tree as a result. The merging is done recursively down the two trees, to ensure that no immediate sibling nodes in the FSS are labeled by the same state. This in turn guarantees that at all times, the branching degree of every node in the FSS is bounded by the number of states in the parsing table, a property essential for maintaining a linear bound on the running time of the algorithm. Two trees may end up having the same top state as a result of either a shift operation or a reduction. In the shift case, since prior to the shift the trees necessarily had different top states, they may be simply merged at the top node level, and no deeper tree contraction is needed. However, in the case of a reduction, if the result of the reduction is a top state which is the same of that of another existing tree in the FSS, a full CONTRACT operation is performed.

The RECLAIM operation is responsible for freeing the dynamically allocated storage for those nodes and trees that are discarded in the course of the algorithm.

\subsection{A Formal Description of the Algorithm}

We next present a more formal description of algorithm $S S R$ in a pseudo "high-level" language.

We use the following notation:

- nodes of the FSS are presented as structures with two fields. A state field containing the parser state, and an action field containing the next parser action to be done upon processing the node.

- actions are of the form Shift(s) in case of a shift, where $s$ is the state of the new node to be created after shifting the input symbol, Goto(s) at the end of a reduction, where $s$ is the state of the new node created after shifting the left-hand side non-terminal symbol of the rule reduced, or of the form Reduce(i) in the case of a reduction, where $i$ is the number of the grammar rule being reduced.

- STATES is the set of all parser states (according to the parsing table).

- ROOTS is the set of nodes that are roots of trees in the FSS. 
- NEW_ROOTS - temporary set of new roots.

- EOS - token representing the end of the input string.

- get_next_sym(x) : function returning the next input token $x$.

- length_of_rule(i) : function returning the length (in number of symbols) of right hand side of grammar rule $i$.

- left_hand_sym(i) : function returning the left hand side non-terminal symbol of grammar rule $i$.

- $A C T(s, s y m):$ Action specified in the parsing table for state $s$ and symbol sym.

- $L O N G(s, s y m)$ : Set of goto states in the long reduction goto table for top state $s$ and symbol sym.

The following is the high level description of the algorithm :

(1) INIT :

get_next_sym $(x)$;

let $S=\left\{s\right.$ in STATES | exists a state $s^{\prime}$ st $\left.\operatorname{ACT}\left(s^{\prime}, x\right)=\operatorname{Shift}(s)\right\}$;

For each $s$ in $S$

do :

create a node $\mathrm{n}$ with $\mathrm{n} . \mathrm{state}=\mathrm{s}$;

add $n$ to ROOTS;

end;

(2) TERM :

get_next_sym( $x)$;

if ROOTS $=\{\}$ then REJECT

else if $x=$ EDS then ACCEPT

else

(3) DISTRIBUTE :

For each node $\mathrm{n}$ in RODTS do $\mathrm{n}$.action $=\operatorname{ACT}(\mathrm{n} . \mathrm{stat} \theta, \mathrm{x})$;

(4) REDUCTION PHASE :

unmark all states for long reductions;

while there exists a node $n$ in ROOTS with n.action $=$ Reduce $(i)$

do :

REDUCE_TREE $(n, i)$;

end;

if (length_of_rule(i) >0) then $\operatorname{ReclaIM}(n)$;

(5) SHIFT PHASE :

NEW_ROOTS $=\{\}$;

for each node $n$ in ROOTS do

if $n$.action $=$ Shift (s) then

if there exists a node $n^{\prime}$ in NEH_ROOTS with $n^{\prime}$.state $=8$ 


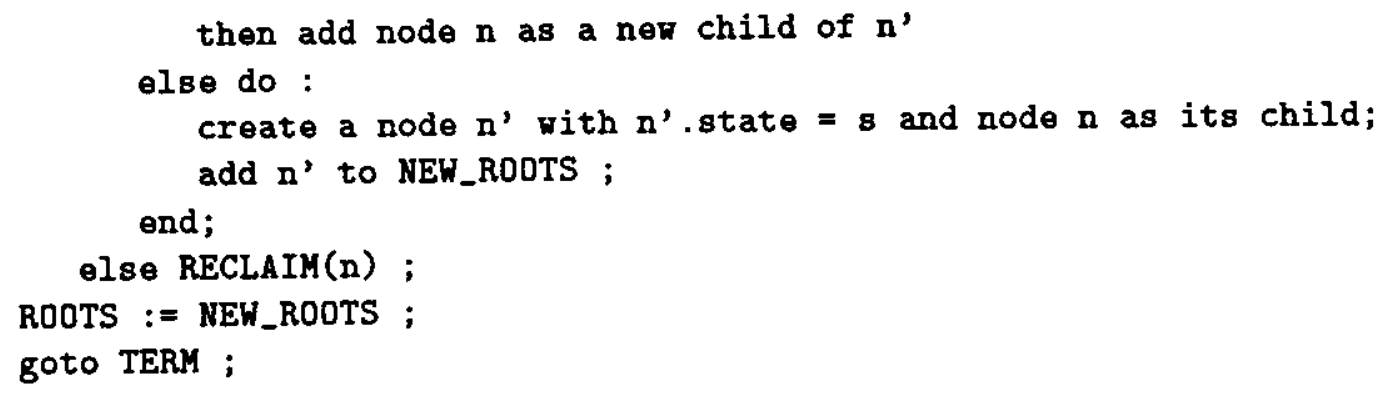

\subsection{Description of Procedures}

\subsubsection{REDUCE_TREE}

REDUCE_TREE performs the reduction operation on an entire tree rooted at a given node $n$, according to a given rule $i$ of the grammar.

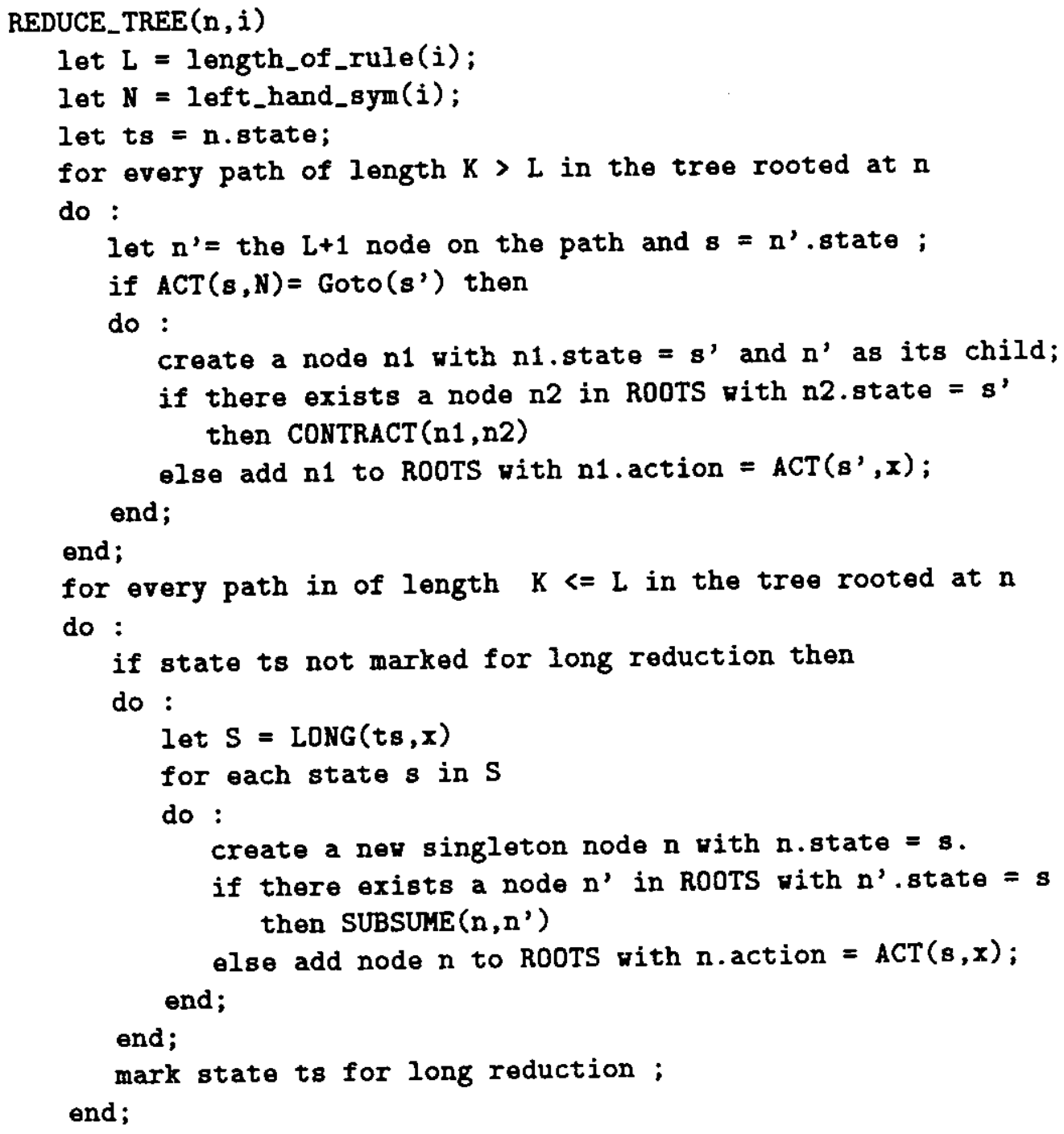




\subsubsection{CONTRACT}

CONTRACT merges two trees that have root nodes of the same state into a single tree.

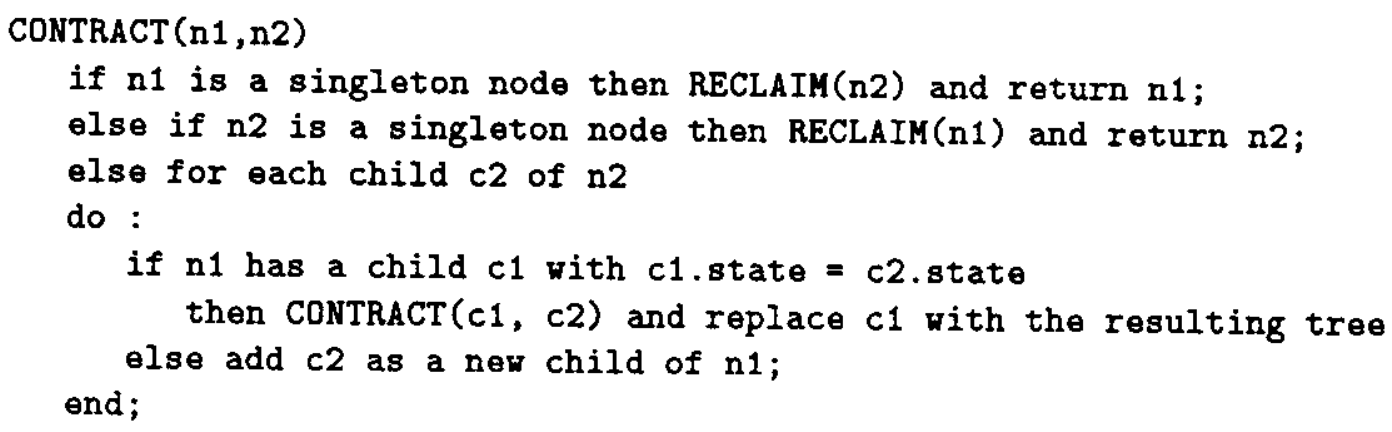

\subsubsection{SUBSUME}

$S U B S U M E$ replaces a tree rooted at a node $n$ with a singleton new node that has the same state.

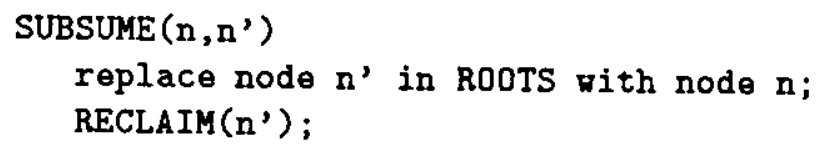

\subsubsection{RECLAIM}

$R E C L A I M$ deletes all nodes of the tree rooted at given node $n$ from the Forest Structured Stack.

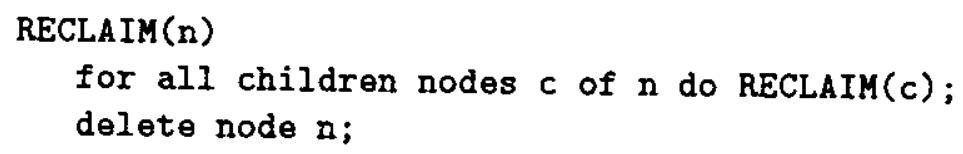

\subsection{An Example}

To further clarify how the algorithm works, we present a simple example. Figure 1 contains a simple arithmetic expression grammar, taken from [ASU86] (page 218). Table 1 contains the SLR parsing table for this grammar, as it appears in Figure 4.31 of [ASU86] (page 219). Table 2 shows the long reduction goto table for this parsing table. For each state, the long reduction goto table contains the list of states into which the parser may shift after a reduction from that state ${ }^{2}$. Figure 2 shows the contents of the FSS along the various stages of the execution of the algorithm on the input "* id )".

Let us follow a trace of this execution. The initialization stage of the algorithm results in entering a single node of state 7 into the FSS, since this is the only state that is the result of shifting the first input symbol " *"'. Thus, after the initialization, the FSS contains the the single node tree shown in Figure 2a. State 7 wishes to shift the next input symbol "id" ", thus the first Reduce Phase is empty, and the shifting of "id" occurs in the Shift Phase, resulting in

\footnotetext{
${ }^{2}$ Note that in the general $\operatorname{LR}(k)$ case, the action table may indicate reductions by several different rules from a particular state for different lookaheads. Thus, strictly speaking, the long reduction goto table specifies a partial function from top states and lookaheads to sets of states. However, in our simple example, the grammar is LR(0) and a reduction by at most a single rule is possible from each state. We have therefore simplified the table for this example by omitting the lookaheads.
} 

1. $E \rightarrow E+T$
2. $\mathrm{E} \rightarrow \mathrm{T}$
3. $\mathrm{T} \rightarrow \mathrm{T} * \mathrm{~F}$
4. $\mathrm{T} \rightarrow \mathrm{F}$
5. $F \rightarrow(E)$
6. $\mathrm{F} \rightarrow$ id

Figure 1: A simple grammar for arithmetic expressions

\begin{tabular}{|c|c|c|c|c|c|c|c|c|c|}
\hline & \multicolumn{6}{|c|}{ Action } & \multicolumn{3}{|c|}{ Goto } \\
\hline State & id & + & $*$ & ( & ) & $\$$ & $\mathrm{E}$ & $\mathrm{T}$ & $F$ \\
\hline 0 & sh5 & & & $\operatorname{sh} 4$ & & & 1 & 2 & 3 \\
\hline 1 & & sh 6 & & & & acc & & & \\
\hline 2 & & r2 & $\operatorname{sh} 7$ & & r2 & $\mathrm{r} 2$ & & & \\
\hline 3 & & r4 & $\mathrm{r} 4$ & & $\mathrm{r} 4$ & $\mathrm{r} 4$ & & & \\
\hline 4 & sh 5 & & & $\operatorname{sh} 4$ & & & 8 & 2 & 3 \\
\hline 5 & & r6 & r6 & & r6 & r6 & & & \\
\hline 6 & sh5 & & & sh4 & & & & 9 & 3 \\
\hline 7 & $\operatorname{sh} 5$ & & & $\operatorname{sh} 4$ & & & & & 10 \\
\hline 8 & & $\operatorname{sh} \theta$ & & & $\operatorname{sh} 11$ & & & & \\
\hline 9 & & $\mathrm{r} 1$ & sh7 & & $\mathrm{r} 1$ & $\mathrm{r} 1$ & & & \\
\hline 10 & & r3 & r3 & & r3 & r3 & & & \\
\hline 11 & & r5 & r5 & & $\mathrm{r} 5$ & r5 & & & \\
\hline
\end{tabular}

Table 1: SLR parsing table for grammar in Figure 1

\begin{tabular}{|c|c|}
\hline Top state & Goto states after reduction \\
\hline 0 & \\
\hline 1 & \\
\hline 2 & 18 \\
\hline 3 & 29 \\
\hline 4 & \\
\hline 5 & 310 \\
\hline 6 & \\
\hline 7 & \\
\hline 8 & \\
\hline 9 & 1 \\
\hline 10 & 2 \\
\hline 11 & 3 \\
\hline
\end{tabular}

Table 2: Long reduction goto table for the parsing table in Table 1 


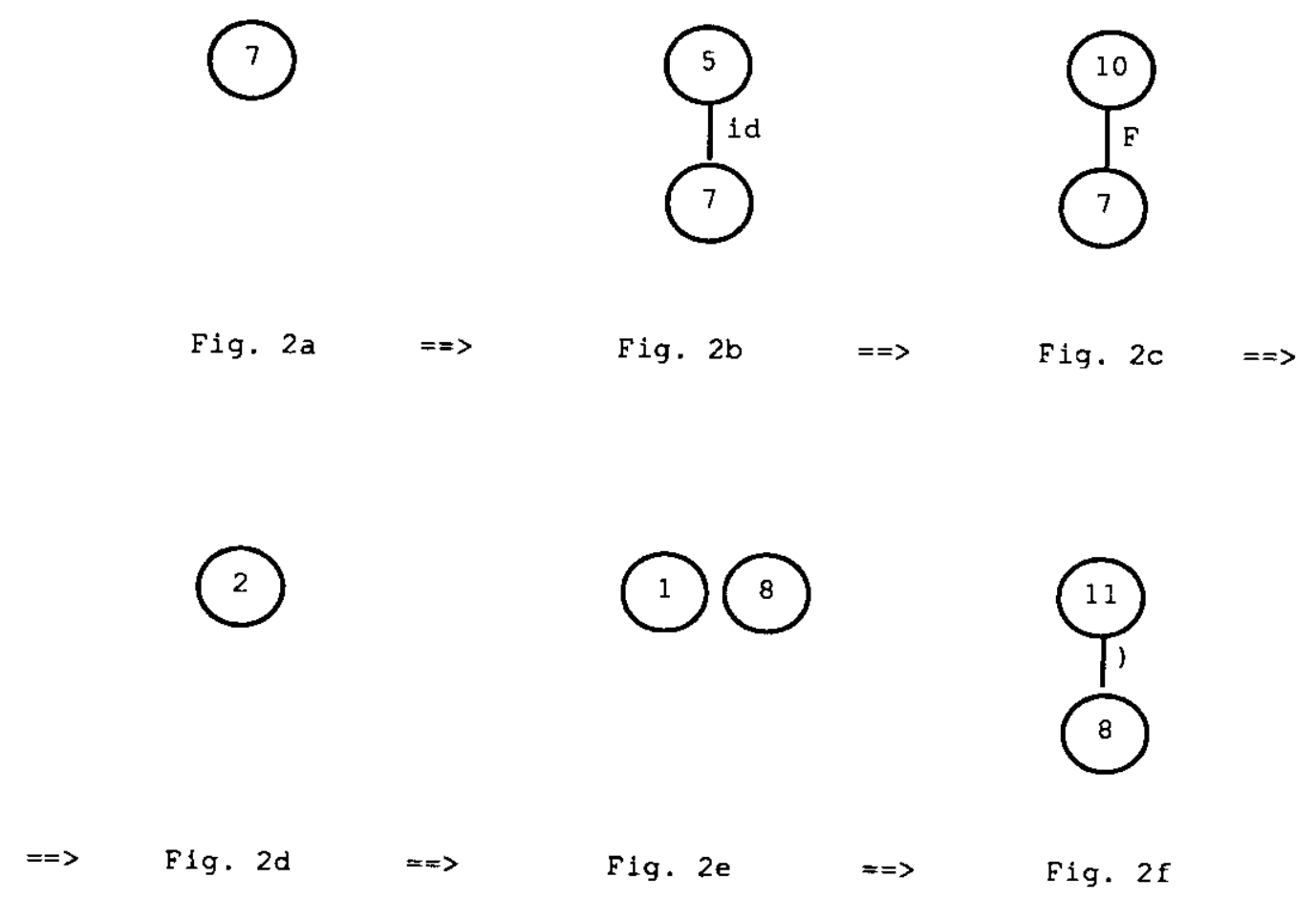

Figure 2: Structure of the FSS throughout the execution of algorithm SSR on the example

the tree in Figure $2 \mathrm{~b}$. The next Reduce Phase includes several reductions. State 5 on input " )", indicates a reduction by rule 6 . This is a normal reduction, and results in the tree in Figure 2c. State 10 on input " ')" indicates a reduction by rule 3 . This is a long reduction. According to the long reduction goto table, this long reduction results in the single node tree of state 2 , as depicted in Figure 2d. State 2 on input " ")" indicates a reduction by rule 2 . This again is a long reduction, and according to the long reduction goto table, it results in two single node trees, of states 1 and 8 respectively, as can be seen in Figure 2e. The Reduce Phase terminates at this point, since neither state 1 nor state 8 indicate a reduction on input " )"'. The following Shift Phase discards the node of state 1 , since the parsing table indicates an error for state 1 on input ' ")". State 8 indicates a shift of ") "' into state 11, resulting in the tree shown in Figure $2 \mathrm{f}$. This completes the Shift Phase. The consequent termination test discovers that we have reached the end of the input. Since the FSS is not empty, the input is a valid substring (of an arithmetic expression in the language of our grammar), and the algorithm terminates. Note that due to the simplicity of the chosen example, no CONTRACT or SUBSUME operations occurred in the execution outlined above.

\section{Correctness}

We now prove the correctness of $S S R$. The reader is referred to Aho and Ullman [AU72] for a comprehensive proof of correctness of the original LR parsing algorithm $L R P$. In our proof, we rely on the correctness of $L R P$, namely that for an LR grammar $G$, given an input string $x, L R P$ accepts $x$ if and only if $x \in L(G)$. We will therefore aim to prove the following theorem :

Let $G$ be an LR(1) grammar and $x$ be an input string. SSR accepts $x$ if and only if there exist strings $y, z$ such that $w=y \cdot x \cdot z$ is accepted by $L R P$. 
We show that $S S R$ simulates the parsing of $x$ by $L R P$ for all possible prefix strings $y$. If upon shifting $x_{n}$, the last input symbol of $x, S S R$ has not rejected $x$, there exists at least one such prefix string $y$, for which $L R P$ has not rejected the input $y \cdot x$ after the shifting of $x_{n}$. The existence of a suffix string $z$, for which $w=y \cdot x \cdot z$ is accepted by $L R P$ is assured by the fact that LR parsers reject inputs as early as possible [AU72]. We now provide a formalization of the above outline.

Definition 1 A stack configuration $c$ is a triple $(s, x, i)$, where $s=\left[s t_{1}, s t_{2}, \cdots, s t_{k}\right]$ is a stack of states (with $s t_{k}$ at the top), $x$ is the input string of length $n$, and $0 \leq i \leq n$ is a position within the input string.

The set of stack configurations represented at any point of $S S R$ includes a configuration for each path from a root node to a leaf in the FSS. The LRP stack configurations are those particular configurations that correspond to stacks manipulated by $L R P$.

Definition 2 A stack configuration $c=\left(s^{\prime}, w, j\right)$ is an $L R P$ stack configuration if after some number of steps of $L R P$ on input $w, s^{\prime}$ represents the $L R P$ stack and $j$ is the parser's position within the input string. In particular, the stack representation $s^{\prime}$ of an $L R P$ stack configuration $c$ always has the LR machine's start state at the bottom of the stack. Formally, we define the function step $(x, k)$ which returns the pair $(s, i)$, where $s$ is the state stack of the parser after $k$ steps on input $x$, and $i$ is the parser's position within the input string $x$. step is uniquely defined by the LR action table. For all $x, \operatorname{step}(x, 0)=\left(\left[s t_{0}\right], 0\right)$, where $s t_{0}$ is the initial state of the LR parser. $c=(s, x, i)$ is an $L R P$ stack configuration if there exists a $k$ such that $\operatorname{step}(x, k)=(s, i)$.

To formally prove that $S S R$ simulates the parsing of the input string $x$ by $L R P$ for all possible prefix strings $y$, we define a mapping function $M$, from general stack configurations to their corresponding $L R P$ stack configurations.

Definition 3 We define the function $M$, from stack configurations to sets of stack configurations in the following way. A stack configuration $c=(s, x, i)$ of the FSS is mapped by $M$ to the (possibly infinite) set of all $L R P$ stack configurations with $s$ as the top portion of the stack. Formally, let $S^{*}$ denote the set of all state stacks, and $L R C$ denote the set of all $L R P$ stack configurations. Then :

$$
M((s, x, i))=\left\{(r \cdot s, y \cdot x,|y|+i) \in L R C \mid r \in S^{*} \& y \in \Sigma^{*}\right\}
$$

where $r \cdot s$ denotes the concatenation of the state stacks. We extend the domain of $M$ to the sets of configurations in the natural way, namely $M\left(\left\{c_{i}\right\}\right)=\bigcup_{i} M\left(c_{i}\right)$.

In the following analysis we assume that all states of the LRP parsing table are reachable from the start state. If in fact this property does not hold, we may easily (in constant time and space) modify the table to include only such reachable states, and use our modified table instead of the original one.

Lemma 1 Let $c_{1}=(s, x, i)$ and $c_{2}=(r \cdot s, x, i)$ be two stack configurations. Then $M\left(c_{2}\right) \subseteq M\left(c_{1}\right)$.

Proof: Straightforward from the definitions of $L R P$ stack configurations and the configuration mapping function $M$.

Both the SUBSUME and CONTRACT operations of $S S R$ remove paths from the FSS when there exist other paths in the FSS that are suffixes of the paths being removed. Lemma 1 implies that the removal of such paths from the FSS does not alter the set of $L R P$ configurations denoted.

To formalize the effect of the parsing operations of algorithm SSR on the FSS, we define the function next, from stack configurations to sets of stack configurations. 
Definition 4 For a given configuration $c=\left(\left[s t_{1}, s t_{2}, \cdots, s t_{l}\right], x, j\right)$, next $(c)$ is the set of configurations $c^{\prime}$ that are the result of a single $S S R$ parsing step from $c$. Thus, as in the $S S R$ algorithm, $n e x t(c)$ is defined according to the action $A C T\left(s t_{l}, x_{j+1}\right)$ indicated in the LR action table. In the case of a shift or a normal reduction, next(c) is a set containing the single resulting new configuration. In case of a long reduction, next $(c)$ is the set of all stack configurations consisting of single state stacks, the states of which one can reach after shifting the left-hand side non-terminal of the rule being reduced, as determined by the long reduction goto table. If the action is accept ${ }^{3}$ or the end of string is reached, we define next $(c)=\{c\}$, and if it is reject (a parse error), then $n \operatorname{ext}(c)=\phi$.

Note that if $c=(s, x, j)$ is an $L R P$ stack configuration, then for some $k$, step $(x, k)=(s, j)$, the action cannot be a long reduction, and therefore next(c) contains a single $L R P$ stack configuration $c^{\prime}$, where $c^{\prime}=\operatorname{step}(x, k+1)$.

To formalize the effect of the Reduce and Shift phases, we define the extension of next to sets of stack configurations in the following way.

Definition 5 Let $C=C_{1} \cup C_{2}$ be a set of stack configurations such that $C_{1}$ contains exactly the stack configurations of $C$ whose top state indicates that the next action is a reduction, and $C_{2}$ is the rest of $C$. If $C_{1} \neq \phi$ then $\operatorname{next}(C)=\left\{c^{\prime} \in \operatorname{next}(c) \mid c \in C_{1}\right\} \cup C_{2}$. If $C_{1}=\phi$ then $\operatorname{next}(C)=\left\{c^{\prime} \in \operatorname{next}(c) \mid c \in C_{2}\right\}$.

Thus, reductions have precedence over other actions.

Based on this extended definition of next we define for every $n \geq 0$ the function next ${ }^{n}$, which is the result of $n$ successive applications of next. Note that a Reduce Phase corresponds to some finite number of applications of next and that a Shift Phase corresponds to a single application of next. Also note that again, if $c=(s, x, j)$ is an $L R P$ stack configuration, then for some $k$, $\operatorname{step}(x, k)=(s, j)$, the action taken on any of the $n$ following parsing steps cannot be a long reduction, and therefore, for any $n \geq 0, n e x t^{n}(\{c\})$ contains the single $L R P$ stack configuration $c^{\prime}$, where $c^{\prime}=\operatorname{step}(x, k+n)$.

Lemma 2 The Simulation Lemma :

Let $C$ be a set of stack configurations. Then : $M(\operatorname{next}(C))=\operatorname{next}(M(C))$

Proof: We prove this by case analysis on the parsing actions that occur on each $c \in C$. The cases of Shift, Accept, Reject and normal reductions are straightforward, as next is identical to the equivalent action of LRP. Long reductions are more subtle, and in this case we show that the result follows from the definitions of $M$ and next :

1. Let $c=(s, x, i)$. Assume $c^{\prime} \in M(n e x t(c)$. Since the action on $c$ was a long reduction, $n \operatorname{ext}(c)$ is a set of configurations of the form $([s t], x, i)$, where the states are determined from the long reduction goto table. Therefore, since st is a reachable state, by the definition of $M, c^{\prime}=(r \cdot[s t], y \cdot x,|y|+i)$ for some state stack $r$ and terminal string $y$, and for some $k, \operatorname{step}(y x, k)=(r \cdot[s t],|y|+i)$. Now the $L R P$ configuration $c^{\prime \prime}=\left(s^{\prime \prime}, y x,|y|+i\right)$ for which step $(y x, k-1)=\left(s^{\prime \prime},|y|+i\right)$ must have $s^{\prime \prime}=r \cdot s$. Therefore, by definition of $M, c^{\prime \prime} \in M(c)$ and $c^{\prime}=\operatorname{next}\left(c^{\prime \prime}\right) \in \operatorname{next}(M(c))$.

\footnotetext{
${ }^{3}$ Notice that in practice the action will never be accept, since the algorithm will have terminated upon reaching the end of the input string. However, we include this case for the sake of completeness.
} 
2. Let $c=(s, x, i)$. Assume $c^{\prime} \in \operatorname{next}(M(c))$. Since the action on $c$ is a reduction, $c^{\prime}$ must be of the form $(r \cdot[s t], y x,|y|+i)$, where the state st is a result of shifting the left-hand side non-terminal at the end of the reduction. From it's definition, the long reduction goto table includes state $s t$ as a possible result of the long reduction on $c$. Thus, $([s t], x, i) \in \operatorname{next}(c)$ and by definition of $M, c^{\prime} \in M(n \operatorname{ext}(c))$.

We generalize Lemma 2 to any finite number of applications of next.

Lemma 3 The Generalized Simulation Lemma :

Let $C$ be a set of stack configurations. For every $n \geq 1: M\left(n e x t^{n}(C)\right)=\operatorname{next}^{n}(M(C))$

Proof: By a straightforward induction on $n$ using Lemma 2.

Base: $i=1:$ Since $n \operatorname{ext}^{1}(C)=n \operatorname{ext}(C)$, by Lemma $2 M\left(n \operatorname{ext}^{1}(C)\right)=n \operatorname{ext}(M(C))$

Induction Hypothesis: Claim is true for all $n<m$.

Induction Step: Proof for $n=m$.

Let $C^{\prime}=n e x t^{m-1}(C)$. By the induction hypothesis we have that

$M\left(C^{\prime}\right)=M\left(n e x t^{m-1}(C)=n e x t^{m-1}(M(C))\right.$. The following set of equalities complete the proof of our claim :

$$
\begin{aligned}
M\left(\operatorname{ext}^{m}(C)\right) & =M\left(n \operatorname{ext}\left(n e x t^{m-1}(C)\right)\right) & & \text { by def. of next } \\
& =M\left(n \operatorname{ext}\left(C^{\prime}\right)\right) & & \text { by def of C' } \\
& =n \operatorname{ext}\left(M\left(C^{\prime}\right)\right) & & \text { by Lemma } 2 \\
& =n \operatorname{ext}\left(n \operatorname{ext}^{m-1}(M(C))\right) & & \text { by induction hyp. } \\
& =n \operatorname{ext}^{m}(M(C)) & & \text { by def. of next }
\end{aligned}
$$

This completes the proof of the Lemma 3.

Lemma 4 When parsing an input string $x=x_{1} x_{2} \cdots x_{n}$, let $C_{1}$ be the set of initial stack configurations of the FSS, and let $C_{i}$ denote the set of stack configurations represented by the FSS after the ith Shift Phase. The following two properties are maintained for each of the $C_{i}(1 \leq i \leq n)$ :

1. Soundness : if $c \in C_{i}$ then $M(c) \neq \phi$

2. Completeness : for all LRP stack configurations $c^{\prime}=(r \cdot s, y x,|y|+i)$, such that the last operation of the parser is a shift of $x_{i}$, there exists a configuration $c=(s, x, i) \in C_{i}$ such that $c^{\prime} \in M(c)$.

Proof: By induction on $i . C_{1}$ has both properties due to the way it is constructed. The induction step is proven by the following arguments. Since the next function is a formal modeling of the Reduce and Shift phases of the algorithm (excluding the process of possibly discarding some configurations by SUBSUME and CONTRACT operations), it follows that for some $n$, $C_{i} \subseteq n e x t^{n}\left(C_{i-1}\right)$ (with the "missing" configurations being those discarded by the SUBSUME and CONTRACT operations) and since SUBSUME and CONTRACT have no effect on the set of configurations represented by $M, M\left(C_{i}\right)=M\left(n e x t^{n}\left(C_{i-1}\right)\right)$. The next function has the property that if $M(c) \neq \phi$ and $n e x t(c) \neq \phi$, then $M(n e x t(c)) \neq \phi$, which extends to next $t^{n}$ and thus guarantees soundness. By Lemma $3 M\left(C_{i}\right)=M\left(n \operatorname{ext}^{n}\left(C_{i-1}\right)\right)=\operatorname{next}^{n}\left(M\left(C_{i-1}\right)\right)$, which guarantees completeness. 
Corollary 1 If $C_{n}$ is the set of stack configunations represented by the FSS after the nth Shift Phase, where $n=|x|$, then $C_{n} \neq \phi$ iff there exists an LRP configuration $c^{\prime}=\left(s^{\prime}, y x,|y|+|x|\right)$.

Note that the existence of such an $L R P$ configuration $c^{\prime}$ implies the existence of a string $w^{\prime}=y x$, such that $w^{\prime}$ is not rejected by $L R P$ by the time $x_{n}$ was shifted. The soundness property of Lemma 4 guarantees that if $C_{n} \neq \phi$, such an $L R P$ stack configuration $c^{\prime}$ exists. The completeness property guarantees that if such a configuration $c^{\prime}$ exists, $C_{n} \neq \phi$.

We may now proceed to proving the main theorem:

Theorem 1 Let $G$ be an LR grammar, and $x$ be a given input string. Algorithm SSR accepts $\mathrm{x}$ if and only if there exist strings $y, z$ such that $w=y \cdot x \cdot z$ is accepted by algorithm LRP.

Proof: 1. If: Since there exist strings $y$ and $z$ such that $w=y \cdot x \cdot z$ is accepted by algorithm $L R P$, the string $w^{\prime}=y \cdot x$ is not rejected by $L R P$ up to the point of the shifting of $x_{n}$ (where $n=|x|$ ). Thus, from the above corollary it follows that the FSS of algorithm $S S R$ is not empty upon entering the nth TERM stage, and $x$ will be accepted by $S S R$.

2. Only if: Algorithm SSR accepts $x$ only if the FSS is not empty upon entering the nth TERM stage. If the FSS is not empty, the above corollary implies that there exists a string $y$ such that $w^{\prime}=y \cdot x$ is not rejected by algorithm $L R P$ up to the point of the shifting of $x_{n}$. Since $L R$ parsers have the property that an input is rejected at the first possible opportunity on a left to right scan of the input string [AU72], this implies that there exists a string $z$ such that $w=w^{\prime} \cdot z=y \cdot x \cdot z$ is accepted by algorithm LRP.

This completes the proof of correctness of algorithm $S S R$.

\section{Complexity Analysis}

\subsection{Complexity Analysis for Grammars Free of Epsilon Rules}

We now prove that $S S R$ runs in linear time for grammars free of epsilon rules. In the next subsection we will demonstrate that $S S R$ maintains a linear running time even in the presence of such rules.

After the initialization of the FSS, the algorithm enters a loop that consists of a termination test for end of input, examining the next input symbol, a Reduce Phase and a Shift Phase. This loop can be executed up to $n-1$ times, until the end of string is reached. The initialization of the FSS that precedes the loop requires only constant time. It involves scanning a column of the LR action table, and the creation of a constant number of root nodes. The termination check also takes constant time. Since there are only a constant number of root nodes (see Lemma 5 below), each Shift Phase involves only a constant number of shift operations and thus takes constant time. However the time cost of each Reduce Phase is not uniform, and varies from one run through the loop to the next. Each Reduce Phase involves some number of Tree Reductions, which are reductions on all paths of an FSS tree to a constant depth. We will show that each such Tree Reduction is completed in constant time and then use an amortized cost evaluation to obtain a linear bound on the total number of Tree Reductions. Finally, we will argue that the total time cost of all SUBSUME, CONTRACT and RECLAIM operations is also at most linear in the length
of the input.

In the following analysis, $S$ denotes the set of states of the parser, and $|S|$ is the size of this set. We distinguish between root nodes of the FSS and internal nodes.

Lemma 5 At any time there is at most a single root node of any given state. 
Proof: The claim holds after the initialization of the algorithm, and throughout Reduce and Shift Phases SSR explicitly checks for root nodes of identical state, and when detected, merges the appropriate trees, using SUBSUME and CONTRACT as necessary.

Lemma 6 The total number of nodes that become internal in the course of execution of the algorithm on a string $x$ of length $n$ is $O(n)$.

Proof: In the case that the grammar is free of epsilon rules, root nodes become internal only as a result of shift operations. Once a node becomes internal, it never again becomes a root node. Thus, the Lemma is a direct result of the fact that the number of root nodes at the start of any Shift Phase is bounded by $|S|$, and there are at most $n$ Shift Phases. Thus the total number of shift operations is $O(n)$.

Lemma 7 No node in the FSS ever has more than $|S|$ children.

Proof: Throughout the algorithm CONTRACT operations are performed whenever necessary so as to maintain this property.

We now concentrate on analyzing the time complexity of Reduce Phases. A normal reduction on a single path of nodes in the FSS is identical to an $L R P$ reduction, and takes constant time. Long reductions are very similar to normal reductions. However, they involve accessing the long reduction goto table in order to determine the possible states that may result from the shifting of the left-hand side non-terminal of the rule being reduced. This table access is done in constant time. New root nodes are created for the resulting states of this process, and each added new node may require a $S U B S U M E$ operation, if there already exists a root node of the same state. This condition can be detected in constant time by a linear scan of the set of root nodes, and need be done only a constant number of times per long reduction, since at most $|S|$ new root nodes may be added. We account for the time spent on the SUBSUME operations separately. Therefore, excluding the time spent on all $S U B S U M E$ operations, a long reduction on a single path requires only constant time. Thus, any reduction, normal or long, on a single path requires only constant time.

A Reduce Phase reduction in $S S R$ operates on a FSS stack tree, and performs the reduction on all paths in the tree that originate at the root node to a depth equal to the number of symbols on the right-hand side of the rule being reduced. Since this is a constant depth, and the fan-out degree of FSS tree nodes is also bounded by a constant, each such Tree Reduction involves only a constant number of reductions (one for each path), each taking constant time. Thus in order to complete the time analysis of Reduce phases, we need only demonstrate that $O(n)$ Tree Reductions are performed in the course of the algorithm.

For the purpose of the analysis, we separate the rules of our grammar into two groups. Grammar rules with a single symbol on the right-hand side are grouped together as non-generative rules and their corresponding reductions are referred to as non-generative reductions. All other rules will be called generative rules and their corresponding reductions generative reductions. We will show that the cost of performing a generative reduction can be charged to internal nodes of the FSS that are discarded by the reduction, and that only a constant number of consecutive non-generative reductions may occur between the generative ones. Thus, the non-generative reductions may be charged to the generative ones, and they in turn can be charged to the nodes.

Lemma 8 In a Reduce Phase of algorithm SSR, only a constant number of consecutive nongenerative Tree Reductions may be performed. 
Proof: Since long reductions are performed at most once per state in a Reduce Phase, we need only consider the normal reductions. Non-generative reductions do not remove internal nodes from the FSS. By a counting argument it can be seen that after a constant number of such reductions on FSS trees, such a reduction is repeated. If this were to occur the non-generative rules that correspond to this series of reductions would form a cycle, in contrast with the fact that any LR grammar must be non-cyclic.

Lemma 9 In the course of an execution of algorithm $S S R$, there are only $O(n)$ generative Tree Reductions.

Proof: We recall that at most one long reduction can occur for each state per Reduce Phase. Therefore, at most $O(n)$ such reductions may occur in all Reduce Phases combined. Thus again we need only consider the normal reductions. Generative normal reductions are performed on trees with internal nodes. Such a Tree Reduction will remove all internal nodes to a depth corresponding to the number of symbols on the right-hand side of the rule. We therefore account for these reductions by charging a unit of cost to each internal node removed by the Tree Reduction. Since the node is removed from the FSS by the Tree Reduction, it may only be charged once. Also, for each generative Tree Reduction performed, at least one internal node is charged. Thus the total number of internal nodes charged is an upper bound on the total number of generative Tree Reductions. By Lemma 6 there are only $O(n)$ nodes that become internal in the course of the execution of $S S R$. Thus, at most $O(n)$ internal nodes may be charged for Tree Reductions and we obtain an $O(n)$ bound on the total number of generative Tree reductions.

Lemma 10 The Total number of Tree Reductions is $O(n)$.

Proof: We look at the non generative reductions as groups of consecutive reductions that occur before, between and after the generative reductions. Due to the $O(n)$ bound on the number of generative reductions, we obtain a similar bound on the number of such groups of non generative reductions. We therefore get an $O(n)$ bound on the total number of non generative Tree Reductions. This in turn provides us with a bound of $O(n)$ on the total number of all Tree reductions.

We complete the time complexity analysis of our algorithm by showing that all CONTRACT, $R E C L A I M$ and $S U B S U M E$ operations together require only $O(n)$ time.

First we consider the CONTRACT operations. The CONTRACT operation merges two FSS trees that have root nodes of the same state. The contraction itself is done by comparing the states of the children of the first root node with those of the second root node. Lemma 7 guarantees at most $|S|^{2}$ comparisons. If a child of the first root node has a state identical to that of a child of the second root node, the two subtrees are contracted by a recursive call to CONTRACT. All other children (and their appropriate subtrees) are added as children of the first root node, and the second root node is deleted. Thus, the top level CONTRACT operation requires constant time. Note that any recursive call to CONTRACT will necessarily result in the elimination of an internal node. We may thus charge a unit of cost to the node deleted as a result of each recursive call to CONTRACT, and since the node is deleted from the FSS by the this operation, it may be charged only once. Since CONTRACT is invoked only after reductions, there are at most $O(n)$ top level calls to CONTRACT. Lemma 6 guarantees that at most $O(n)$ internal nodes will be charged, therefore implying at most $O(n)$ recursive calls to CONTRACT. This provides us with an $O(n)$ 


\section{1. $S \rightarrow a S b$ \\ 2. $S \rightarrow \epsilon$}

Figure 3: An LR(0) grammar for the language $a^{n} b^{n}$

bound on the total number of CONTRACT calls and a similar bound on the total time complexity of all CONTRACT operations.

Next, we consider the RECLAIM operations. These operations delete entire subtrees from the FSS, when these become obsolete. We assume the cost of such an operation is directly proportional to the number of nodes in the subtree, i.e. constant time per node being deleted. The deletion of internal nodes can be charged a unit of cost to the node being deleted, and only $O(n)$ root nodes are reclaimed in all Shift Phases and Reduce Phases combined, since at most one root node is reclaimed per operation.

Finally, we observe that we have already accounted for the $S U B S U M E$ operations. SUBSUME searches for a root node of a state identical to that of a new single node tree created by a long reduction. This requires constant time. If found, the tree is the reclaimed by the RECLAIM operation, the time for which we have already accounted for.

This completes the time complexity analysis of our algorithm, under the assumption that the grammar contains no epsilon rules. Our analysis has shown that the total time cost of all operations in an execution of the algorithm on an input string of length $n$ is $O(n)$.

\subsection{Extending the Complexity Analysis to Grammars with Epsilon Rules}

We now turn to deal with the case that the grammar contains epsilon rules. Epsilon rules complicate our algorithm due to the fact that root nodes may become internal nodes as a result of a reduction by an epsilon rule. Thus, Lemma 6 must be re-argued, namely that the total number of root nodes that become internal in the course of an execution of the algorithm continues to be $O(n)$, even in the presence of epsilon reductions.

Since epsilon rules have no effect on the Shift Phase of our algorithm, in order for our entire complexity analysis to still carry through, we need only to prove that the total number of Tree Reductions is still $\mathbf{O}(\mathbf{n})$.

Let us note that a grammar may indeed have epsilon rules, and still be LR. For example consider the natural grammar for the language $a^{n} b^{n}$ (for $n \geq 0$ ) in figure 3 , which is in fact LR(0).

It is convenient to look at epsilon rules as normal grammar rules that generate an "invisible" terminal symbol epsilon. Thus strings in the language generated by the grammar correspond to modified strings that include the epsilon symbols in the appropriate places. For a non-ambiguous grammar we are guaranteed that this is a one to one correspondence (each string in the language corresponds to exactly one string with epsilon symbols).

Lemma 11 An LR grammar has the property that only a constant number of epsilons may appear between two non-epsilon terminal symbols in the modified strings that correspond to strings in the language generated by the grammar. Furthermore, if we denote the length of the longest right hand side of all grammar rules by $L$, and the number of grammar rules by $i$, this constant number of consecutive epsilons is bounded by $L^{i}$.

Proof: In order to prove this claim we restrict our attention to $E$, the subset of grammar rules that may produce a consecutive string of epsilons. It is easy to see that if the rules in $E$ 
can produce an infinite string of epsilons (starting from any rule in $E$, whose left-hand side non-terminal is reachable), then the grammar is necessarily ambiguous and thus not LR. The fact that $E$ cannot produce an infinite string of epsilons poses several restrictions on the rules in this subset. No rule in $E$ contains a terminal symbol on its right-hand side. Also, no rule in $E$ can be recursive (the left-hand side non-terminal cannot appear on the right-hand side of the rule). Using these properties, by a simple induction on $i$, the number of rules in $E$, it can be shown that the number of consecutive epsilons that can be produced by $E$ is bounded by the constant $C_{e}=L^{i}$, where $\mathrm{L}$ is the length of the longest right-hand side of the rules in
$E$.

In order to prove that the total number of Tree Reductions continues to be $O(n)$, it is sufficient for us to show that Lemma 6 still holds.

Lemma 12 The total number of root nodes that become internal nodes in the course of an execution of algorithm $S S R$ on a string $x$ of length $n$ is $O(n)$, even if the grammar has epsilon rules.

Proof: For every i: $0<i \leq n$, let internal $(i)$ be the total number of nodes that have become internal in the course of the algorithm, up until the completion of the Shift Phase of $x_{i}$. We prove by induction on $i$ that for every $0<i \leq n$, internal $(i) \leq C * i$, where $C$ is a the constant $|S| *\left(C_{e}+1\right)$.

Base : $i=1$ : The shifting of $x_{1}$ occurs in the INIT stage, where at most $|S|$ root nodes are created. Since no internal nodes exist at this point, the claim is trivially true.

Induction Hypothesis: Assume the claim is true for $i \leq m$.

Induction step : Proof for $i=m+1$. Clearly internal $(m+1)$ is the sum of internal $(m)$ and the number of root nodes that became internal during the Reduce Phase of $x_{m}$, and the Shift phase of $x_{m+1}$. from the analysis presented above, it is clear that during the Reduce Phase, at most $C_{e}$ epsilon reductions can be performed on each individual tree of the FSS (corresponding to the maximum number of epsilons that may appear in the string prior to the next "real" terminal symbol). Since each such epsilon reduction causes a single root node of the FSS to become internal, the total number of root nodes that become internal during the Reduce Phase due to epsilon reductions is bounded by $|S| * C_{e}$. As before, in the consequent Reduce Phase of $x_{m+1}$, since there are at most $|S|$ root nodes upon entering the Shift Phase, at most $|S|$ root nodes may become internal nodes by the end of the Shift Phase. Summing up the total number of nodes that become internal, we get :

$$
\begin{array}{rlr}
\text { internal }(m+1) & \leq \text { internal }(m)+|S| * C_{e}+|S| & \\
& \leq C * m+C & \text { (by induction hypothesis) } \\
& =C *(m+1)
\end{array}
$$

Now since the total number of nodes that become internal in the course of the execution of algorithm $S S R$ is bounded by internal $(n)$, and internal $(n) \leq C * n$, the above total has indeed been shown to be $O(n)$.

In the process of proving the above lemma, we in fact have shown that only $O(n)$ epsilon reductions may occur in the course of executing $S S R$ on a string $x$ of length $n$. It thus follows that Lemma 10 continues to hold, and the number of Tree Reductions continues to be $O(n)$, taking into account all three types of tree reductions that now exist, non-generative tree reductions, generative tree reductions, and epsilon rule tree reductions. Combined with the time analysis of the other operations which continues to hold as before, we may again conclude a linear time bound on the total running time of algorithm $S S R$. 


\section{The Algorithm for Canonical LR(k) Grammars}

In this section we consider the implications of generalizing algorithm $S S R$ to deal with the general case of canonical LR(k) parsing tables.

First, let us consider the necessary modifications to the algorithm itself. These turn out to be quite minimal. In fact, only the INIT stage needs to modified. In the INIT stage, instead of reading just the first symbol of the input string, we must obtain the first $k$ symbols for the lookahead. This is due to the fact that the $\operatorname{LR}(\mathrm{k})$ action table is defined according to the $k$-lookahead on the input. The action table is then searched in order to construct the initial set of root nodes. An obvious complication occurs whenever the length of the input string is less than the needed lookahead $(|x|<k)$. To handle this case, all possible extensions of the input string $x$ to a string $y$ of length $k$ are considered, and the set of root nodes is constructed as the union of the sets derived for all such $y$. The algorithm will then terminate immediately in the following TERM stage. If the set of root nodes constructed in the INIT stage is not empty, $x$ is accepted, otherwise $x$ is rejected.

Following is the "high level" description of the modified INIT stage :

- first_k_syms(x) returns the first $k$ symbols of the input string $x$. If $|x|<k$ it returns the entire string $x$.

(1) INIT :

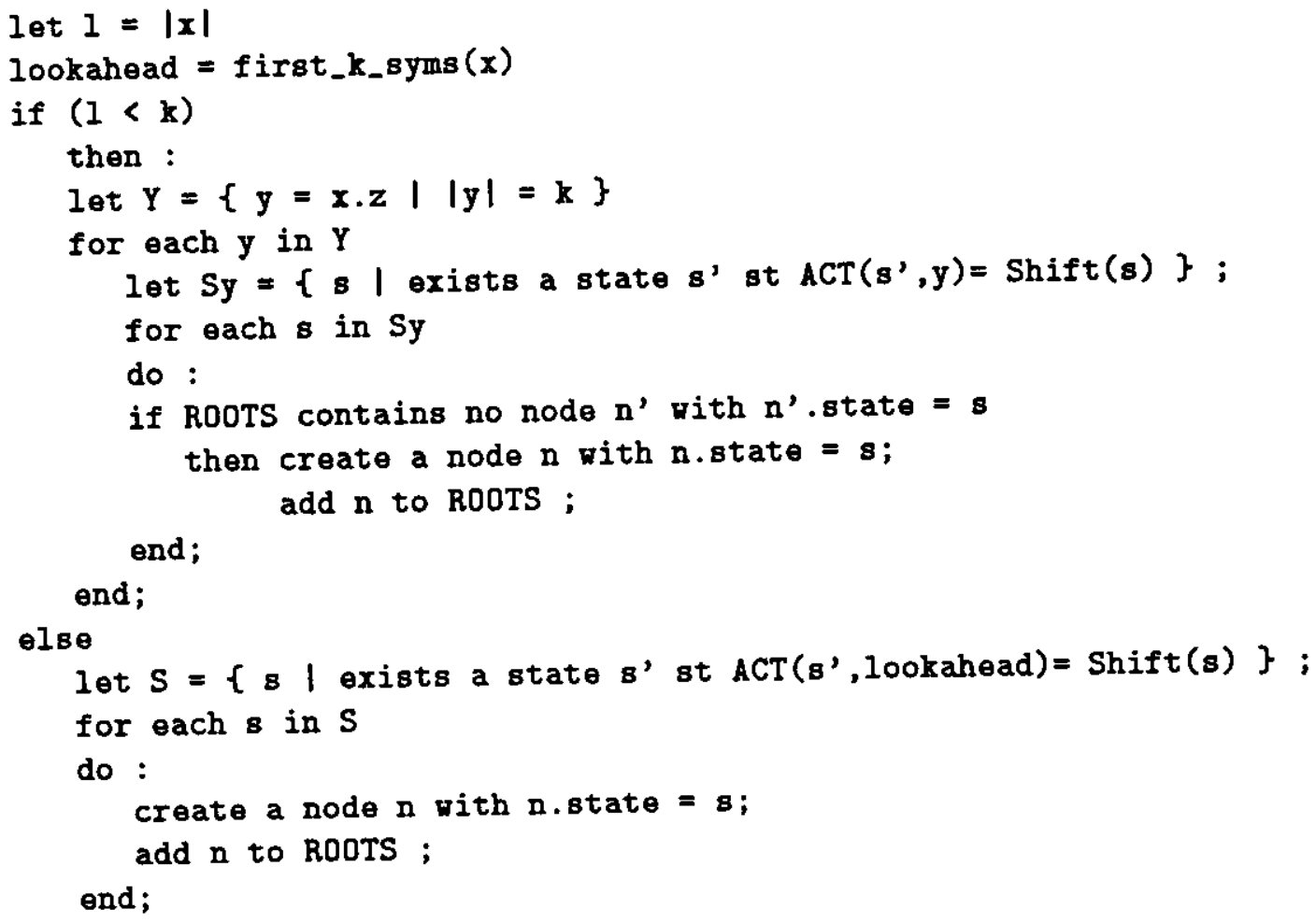

All other stages of the algorithm stay exactly the same as in algorithm $S S R$, as presented in section 2. In the DISTRIBUTE stage, the actions determined from the LR(k) action table depend on the existing $k$-lookahead at that particular point in time. In the Shift Phase, the first symbol of the lookahead (the symbol being shifted) is removed from the lookahead and shifted. The get_next_sym function call in the subsequent TERM stage completes the lookahead from length $k-1$ to $k$. The algorithm terminates when the end of string (EOS) is encountered, with $k-1$ symbols of the input string still in the lookahead. 
Let us now consider what implications (if any) does the above modification of algorithm $S S R$ have on its correctness and complexity.

The proof of correctness presented in section 3 continues to hold for our modified algorithm. Lemma 4 continues to hold with respect to the appropriate $\operatorname{LR}(\mathrm{k})$ version of algorithm $S S R$. Since the property of rejecting an input at the earliest possible opportunity [AU72] holds for general LR(k) grammars, the proof of the main theorem of correctness continues to hold as well.

Finally let us consider the complexity analysis. It is easily seen that the revised INIT stage still takes only constant time. The set $S y$ is a finite set bounded by a constant, thus constructing the initial set of root nodes clearly takes only constant time. The size of this set is still bounded by $|S|$, the number of states in the LR action table. Since all other stages of algorithm $S S R$ are the same as before, the time complexity analysis of the algorithm remains valid.

\section{Conclusions}

We have presented and proved a linear time algorithm for recognizing substrings of LR(k) languages.

The original version of this algorithm was initially developed by the first author in 1980 . It did not include the CONTRACT operation for merging trees of the FSS. Tree contractions are crucial to retaining a linear bound on the running time of the algorithm. In the process of trying to prove the linear time bound we discovered this deficiency, and the proper modifications were consequently made.

The original algorithm, while in fact not always linear, was used as the basis for a syntax checking modification to the IBM VM/370 editor XEDIT. That modification enabled the IBM editor to check COBOL source code for syntax errors, when users modified lines, screens or files. For instance, when the cursor was moved off a modified line, the editor would beep and display an unobtrusive error message if the line was not a substring of any COBOL program. Though COBOL has a large grammar, this modification had no apparent effect on the speed of XEDIT on machines of the early 1980's. The algorithm was also used to check Pascal programs on an IBM PC editor, and this too had no apparent effect on the speed of the editor. Thus, the original algorithm appeared to be adequately fast in practice.

We have implemented our revised algorithm and have tried it on several test grammars. No precise measurements have been performed to compare the actual running time of our substring algorithm with that of the original LR parser. However, in practice, the revised implementation
continues to run as fast as before.

\section{Acknowledgements}

Alan Demers provided helpful discussion during development of the original algorithm and proof sketches in 1979. Merrick Furst was helpful in the development of some of the revised ideas described here. We also thank Steve Guattery for his comments.

\section{References}

[AD83] R. Agrawal and K.D. Detro. An efficient incremental LR parser for grammars with epsilon
productions. Acta Informatica, 19:369-376, 1983.

[AJ74] A. V. Aho and S.C. Johnson. LR parsing. Computing Surveys, 6(2):99-124, 1974. 
[ASU86] A. V. Aho, R. Sethi, and J. D. Ullman. Compilers: Principles, Techniques and Tools. Addison-Wesley, 1986.

[AU72] A. V. Aho and J. D. Ullman. The Theory of Parsing, Translation and Compiling, Vol. I: Parsing. Prentice-Hall, Englewood Cliffs, N.J., 1972.

[AU77] A. V. Aho and J. D. Ullman. Principles of Compiler Design. Computer Science and Information Processing. Addison-Wesley, 1977.

[Cel78] A. Celentano. Incremental LR parsers. Acta Informatica, 10:307-321, 1978.

[Cor89] G. V. Cormack. An LR substring parser for noncorrecting syntax error recovery. In Proceedings of the SIGPLAN'89 Conference on Programming Language Design and Implementation, pages 161-169, 1989.

[CW87] D. Coppersmith and S. Winograd. Matrix multiplication via arithmetic progressions. In Proceedings of STOC'87, pages 1-6. ACM press, 1987.

[DeR69] F.L. DeRemer. Practical translators for LR(k) languages. Ph.D. dissertation, MIT, Cambridge, Ma., 1969.

[DeR71] F.L. DeRemer. Simple LR(k) grammars. Communications of the ACM, 14(7):453-460, 1971.

[Ear70] J. Earley. An efficient context-free parsing algorithm. Communications of the $A C M$, 13(2):94-102, 1970.

[HU79] J. E. Hopcroft and J. D. Ullman. Introduction to Automata Theory, Languages, and Computation. Computer Science. Addison-Wesley, 1979.

[Knu65] D. E. Knuth. On the translation of languages from left to right. Information and Control, $8(6): 607-639,1965$.

[Kor69] A. J. Korenjak. A practical method for constructing LR(k) processors. Communications of the ACM, 12(11):613-623, 1969.

[Lin70] G. Lindstrom. The design of parsers for incremental language processors. In Proceedings of Second ACM Symposium on Theory of Computation, pages 81-91, Northampton, Mass., 1970.

[Ric85] H. Richter. Noncorrecting syntax error recovery. ACM Transactions on Programming Languages and Systems, 7(3):478-489, July 1985.

[RK91] J. Rekers and W. Koorn. Substring parsing for arbitrary context-free grammars. In Proceedings of Second International Workshop on Parsing Technologies, pages 218-224, Cancun, Mexico, 1991.

[Tom86] M. Tomita. Efficient Parsing for Natural Language. Kluwer Academic Publishers, Hingham, Ma., 1986.

[Val75] L. Valiant. General context free recognition in less than cubic time. Journal of Computer and Systems Sciences, 10(2):308-315, 1975. 